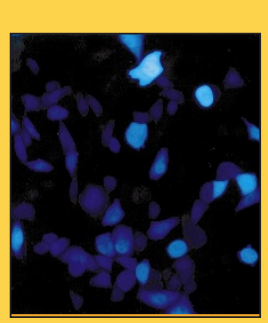

The tetracycliner e p r e s s ible (TetOFF) system has found widespread applications in protein pharmaceutical production and gene therapy research, as an efficient and nontoxic means of controlling transgene expression in mammalian cells. One limitation, however, has been the lack of satisfactory ways of independently controlling expression of a second transgene. In this issue, such a versatile and complementary system regulated by the streptogramin family of human oral antibiotics. They combined a bacterial promoter element $\left(\mathrm{P}_{\mathrm{PTR}}\right)$ and a pristinamycin repressor, the Pip protein, with various promoters and viral transactivation domains, to create both streptogramin-repressible and -inducible systems that can work in combination with TetOFF in a variety of human cell lines (see p. 1203 ).

ND Fussenegger and colleagues have developed

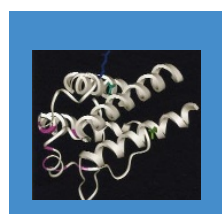

Recombinant interleukin 2 (IL-2) is effective in metastatic cancer; however, for many patients its use is limited by severe systemic toxicity. In this issue, Shanafelt and colleagues have developed an IL-2 variant, called BAY50479 , that has significantly reduced toxicity while retaining potent therapeutic effects. Some evidence has suggested that IL-2's therapeutic effects are mediated by T-cell activation and its toxic effects by natural killer (NK) cell activation, by IL-2 binding to different forms of the IL-2 receptors residing on their surface. These findings have prompted the researchers to eliminate IL-2 residues that interact with the NK-cell receptor in order to reduce IL-2's toxic effects. The BAY50-479 variant that they produced exhibited a $\sim 3,000$ fold selectivity for T cells over NK cells in vitro. They went on to test its in vivo effects and found that its ability to inhibit tumor metastasis of IL-2; however its toxicity in chimpanzees was greatly reduced (see p. 1197). ND treating advanced-stage in a mouse model was comparable to that

\section{Review}

Crop plants show great promise as alternatives to more traditional expression systems such as mammalian cells for the production of biopharmaceuticals. Most of the major crops are now amenable to transformation, and increasing knowledge about promoters and targeting sequences is allowing transgene expression to be specifically targeted to storage tissues for easy storage and isolation. Compared with mammalian production systems, plants produce high yields of protein with similar glycosylation levels at a fraction of the cost. Seed-specific expression often results in significant yields of stable protein, reducing shipping and storage costs. Expression in tubers or fruits should also facilitate the production of edible vaccines (see, for example, pp. 1167 -Mason). Problems remain, however, with transgene silencing, and more information on mRNA stability is required to achiever higher/consistent levels of expression. On page 1151, Giddings et al. summarize current progress in the production of protein and peptide pharmaceuticals in plants, outlining the main types of expression systems and products currently under development.

$A M$

\section{Technical Reports}

In this issue Esser et al. demonstrate that a histone-like protein from the hyperthermostable eubacterium Thermotoga maritima can be used to enhance transfection efficiency in various cell lines, as compared with several commercially available reagents, with less toxicity. They also demonstrate that the protein can enhance the efficiency of another transfection method, lipofection (see p. 1211).

MS

On page 1213, Stoerker and colleagues present a means of genotyping single-nucleotide polymorphisms by MALDI-TOF mass spectrometry. They use nuclease selections for genotyping by combining the DNA to be analyzed with oligonucleotide probes representing known genotypes. Target-bound, complementary probes are refractory to nuclease attack, and their peaks remain in mass spectra. They demonstrate the approach by genotyping PCR-amplified patient DNA from a region of the human cystic fibrosis transmembrane regulatory gene (CFTR). The technique offers advantages in the speed and cost effectiveness of analysis.

\title{
TNF homes in on tumor vessels
}

Tumor necrosis factor $\alpha$ (TNF) has been shown to have potent antitumor activity, but its clinical use as an anticancer drug is hampered by its severe systemic toxicity. In this issue Curnis et al. (see p.1185) describe the generation of a modified TNF molecule bearing an $\mathrm{N}$-terminal peptide that targets it to CD13, a cell surface molecule expressed on tumor vessels. The modified molecule induced stronger antitumor effects in vivo than TNF, at significantly lower doses, decreasing the tumor burden in lymphoma and melanoma animal models. Thus targeted delivery of TNF may enhance its therapeutic properties. MS

\section{Profiling plant metabolites}

On page 1157 Fiehn et al. describe a new tool for globally assessing the metabolic makeup of plants-a high-throughput technique that will complement genomic and proteomic initiatives. In the report they used gas chromatography/mass spectrometry (GC/MS) to follow the relative concentration of hundreds of metabolites in extracts from various Arabidopsis thaliana genotypes to better understand the effects of genetic variations on metabolism. The results allowed a meaningful comparative analysis of the genotypes. Data mining tools, such as principal component analysis, enabled them to assign metabolic phenotypes using the datasets (see also p. 1142).

MS

\section{Next Month in: Nature Biotechnology}
Whole genome microarrays

A global network of
protein interactions

Producing insecticidal crystals in chloroplasts 